\title{
Quantitative Proteomics Reveal ATM Kinase-dependent Exchange in DNA Damage Response Complexes
}

\author{
Serah Choi ${ }^{\dagger} \ddagger, \S$, Rohith Srivas $\|$, Katherine Y. Fu§ ${ }^{\S}$ Brian L. Hood ${ }^{\perp}$, Banu Dost $^{\#}$, Gregory A. \\ Gibson $^{\nabla}$, Simon C. Watkins ${ }^{\nabla}$, Bennett Van Houten ${ }^{\circ}$, Nuno Bandeira ${ }^{\#}$, Thomas $P$. \\ Conrads $^{\perp}$, Trey Ideker $\|, \#, \bullet$, and Christopher J. Bakkenist ${ }^{\S, \circ, *}$ \\ †Medical Scientist Training Program, University of Pittsburgh School of Medicine, Pittsburgh, \\ Pennsylvania, United States \\ ‡Molecular Pharmacology Graduate Program, University of Pittsburgh School of Medicine, \\ Pittsburgh, Pennsylvania, United States \\ $\S$ Department of Radiation Oncology, University of Pittsburgh School of Medicine, Pittsburgh, \\ Pennsylvania, United States \\ "Department of Bioengineering, University of California San Diego, La Jolla, California, United \\ States \\ ${ }^{\perp}$ Women's Health Integrated Research Center at Inova Health System, Gynecologic Cancer \\ Center of Excellence, Department of Defense, Annandale, Virginia, United States \\ \#Department of Computer Science and Engineering, Skaggs School of Pharmacy and \\ Pharmaceutical Sciences, University of California San Diego, La Jolla, California, United States \\ $\nabla$ Department of Cell Biology and Physiology, University of Pittsburgh School of Medicine, \\ Pittsburgh, Pennsylvania, United States \\ 'Department of Pharmacology and Chemical Biology, University of Pittsburgh School of \\ Medicine, Pittsburgh, Pennsylvania, United States \\ - Department of Medicine, University of California San Diego, La Jolla, California, United States
}

\section{Abstract}

ATM is a protein kinase that initiates a well-characterized signaling cascade in cells exposed to ionizing radiation (IR). However, the role for ATM in coordinating critical protein interactions and subsequent exchanges within DNA damage response (DDR) complexes is unknown. We combined SILAC-based tandem mass spectrometry and a subcellular fractionation protocol to interrogate the proteome of irradiated cells treated with or without the ATM kinase inhibitor KU55933. We developed an integrative network analysis to identify and prioritize proteins that were responsive to KU55933, specifically in chromatin, and that were also enriched for physical interactions with known DNA repair proteins. This analysis identified 53BP1 and annexin A1 (ANXA1) as strong candidates. Using fluorescence recovery after photobleaching, we found that the exchange of GFP-53BP1 in DDR complexes decreased with KU55933. Further, we found that ANXA1 knockdown sensitized cells to IR via a mechanism that was not potentiated by KU55933.

\footnotetext{
(C) 2012 American Chemical Society

*Corresponding Author: Tel: +1-412-623-7765. Fax: +1-412-623-7761. bakkenistcj@upmc.edu.

The authors declare no competing financial interest.

Supporting Information

Figures showing immunoblot detection and an MA plot; tables showing the peptides quantified, the enriched networks, proteins from the chromatin fraction, and enrichment ratios. This material is available free of charge via the Internet at http://pubs.acs.org.
} 
Our study reveals a role for ATM kinase activity in the dynamic exchange of proteins in DDR complexes and identifies a role for ANXA1 in cellular radioprotection.

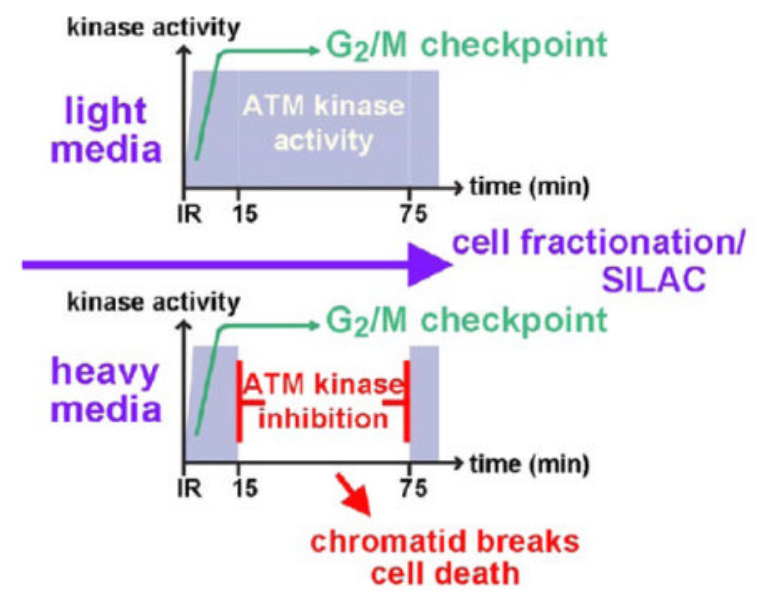

\section{Keywords}

DNA damage response; ATM; 53BP1

\section{INTRODUCTION}

The DNA damage response (DDR) is a complex network of signal transduction pathways that orchestrates a broad array of cellular processes. ${ }^{1}$ Central to the DDR is ATM (ataxia telangiectasia mutated), a serine/threonine protein kinase that is activated by DNA doublestrand breaks (DSBs). ${ }^{2}$ Global proteomic studies have identified over 1,000 substrates of ATM and the related kinase ATR (ATM and Rad3-related), which is activated by singlestranded DNA. ${ }^{3-5}$ These phosphorylations are implicated in diverse mechanisms including cell cycle arrest, DNA replication, and DNA repair. Since ataxia telangiectasia patients are profoundly radiosensitive, there is considerable interest in the development of ATM kinase inhibitors to increase the efficacy of radiotherapy. ${ }^{6}$ To facilitate the development of clinical protocols employing ATM kinase inhibitors, we have investigated the temporal and spatial requirements for ATM kinase activity in irradiated cells.

To investigate the temporal requirement for ATM kinase activity following ionizing radiation (IR), we developed the use of KU55933, a selective and reversible ATM kinase inhibitor, to transiently inhibit ATM kinase activity in cells. ${ }^{7}$ We showed that ATM kinase inhibition for $1 \mathrm{~h}$ following IR abrogated sister chromatid exchange (SCE), increased persistent chromatid aberrations, and increased cell death. ${ }^{7,8}$ Significantly, we also showed that ATM kinase inhibition during this interval affected neither the activation nor the recovery of the $\mathrm{G}_{2} / \mathrm{M}$ cell cycle checkpoint, and also affected neither the accumulation nor the resolution of 53BP1, a protein that mediates ATM kinase signaling, in DDR complexes visualized in fixed cells. ${ }^{8}$ Thus, ATM kinase activity must be sustained after immediate signaling to the $\mathrm{G}_{2} / \mathrm{M}$ cell cycle checkpoint apparatus and after the formation of DDR complexes to mediate mechanisms that ensure DNA repair and cellular radioprotection.

Another potential level of regulation, which has not been well explored, is a role for ATM kinase in coordinating critical interactions and subsequent protein exchanges within DDR complexes. Recent data demonstrate that 53BP1 promotes the repair of DSBs by nonhomologous end-joining (NHEJ) via a mechanism wherein 53BP1 physically impedes 
homologous recombination repair (HRR). ${ }^{9}, 10$ BRCA1 may promote HRR at least in part by removing 53BP1 from sites of damage where it functions to promote DSB repair through NHEJ. ${ }^{9,10}$ Since $53 \mathrm{BP} 1$ is a physical impediment to HRR, it may protect cancer cells with acquired mutations that compromise HRR catalysis by preventing DSB repair through defective HRR pathways. If this is indeed the case, then the removal of the 53BP1 function may drive DSB repair through defective HRR pathways, causing an accumulation of DSB repair intermediates and cell death. Thus, mechanisms that mediate the dynamic exchange of 53BP1 are of interest, since pharmacological disruption of 53BP1 may kill cancer cells that are unable to complete HRR. ${ }^{11}$

To investigate the role of ATM kinase activity in spatial protein dynamics, both in the coordination of critical interactions and subsequent exchanges within DDR complexes, and in the movement of proteins between different cellular compartments, we combined stable isotope labeling by amino acids in cell culture (SILAC)-based tandem mass spectrometry (MS) and a subcellular fractionation protocol to compare the proteome of cells treated with and without KU55933 for $1 \mathrm{~h}$ following IR. We developed an integrative network analysis to identify and prioritize proteins that were responsive to KU55933 specifically in chromatin and were also enriched for physical interactions with known DNA repair proteins. This analysis identified 53BP1 and annexin A1 (ANXA1) as strong candidates. Using fluorescence recovery after photobleaching (FRAP), we found that the exchange of GFP-53BP1 in DDR complexes decreased with KU55933. ANXA1 is a membrane binding protein that has been implicated in membrane trafficking and reorganization. ${ }^{12,13} \mathrm{We}$ found that ANXA1 knockdown sensitized cells to IR via a mechanism that was not potentiated by KU55933.

\section{MATERIALS AND METHODS}

\section{Cell Culture}

IMR90 cells and U2OS cells were cultured in DMEM while H460 cells were cultured in RPMI, both supplemented with $10 \%$ fetal bovine serum (FBS) and penicillin/streptomycin. For SILAC, IMR90 cells were grown in DMEM supplemented with 10\% FBS dialyzed with a cutoff of $10 \mathrm{kDa}$ (Pierce, Rockford, IL). IMR90 were cultured either in "light" SILAC medium (supplemented with L-lysine and L-arginine with natural abundance isotopes) or in "heavy" SILAC medium (supplemented with ${ }^{13} \mathrm{C}_{6}$-lysine and ${ }^{13} \mathrm{C}_{6}{ }^{15} \mathrm{~N}_{4}$-arginine) (Pierce, Rockford, IL) for $>6$ passages to differentially label proteins with isotopes that can be distinguished using mass spectrometry (Figure 1A). ${ }^{14,15}$ Growth in heavy media had no effect on ATM kinase signaling in cells following IR (Supporting Information Figure 1A), as previously shown. ${ }^{3}$

\section{Cell Fractionation}

IMR90 cells cultured in "light" and "heavy" medium were plated with equal cell numbers into three $60 \mathrm{~mm}$ Petri dishes ( 6 total dishes). The next day, both cell populations were exposed to 2 Gy $\gamma$-rays in a Shepherd Mark I model $68\left[{ }^{137} \mathrm{Cs}\right]$ irradiator (J.L. Shepherd \& Associates, San Fernando, CA) at a dose rate of $78.3 \mathrm{R} / \mathrm{min}$. At $15 \mathrm{~min}$ post-IR, $10 \mu \mathrm{M}$ KU55933 ${ }^{16}$ (KuDOS Pharmaceuticals) was added to the cells grown in "heavy" media. At 75 min post-IR, cells were washed twice with PBS, and cells from both "heavy" and "light" populations were pooled and fractionated as described previously. ${ }^{17-19}$

Briefly, $150 \mu \mathrm{L}$ of Solution A (10 mM HEPES (pH 7.9), $10 \mathrm{mM} \mathrm{KCl,} 1.5 \mathrm{mM} \mathrm{MgCl} 2,0.34$ M sucrose, $10 \%$ glycerol, $0.1 \%$ triton X-100) was added to each $60 \mathrm{~mm}$ dish from the "heavy" and "light" populations, and the cells were scraped and collected into the same tube (1:1 ratio) and incubated for $5 \mathrm{~min}$ on ice. Cells were centrifuged at $1300 \mathrm{~g}$, and the 
supernatant was collected as the cytoplasmic fraction. The remaining pellet was washed in Solution A, resuspended in $400 \mu \mathrm{L}$ of Buffer MN (Solution A with $1 \mathrm{mM} \mathrm{CaCl}_{2}$ ) containing 4 units of micrococcal nuclease (Worthington Biochemical Corporation, Lakewood, NJ), and incubated at $37^{\circ} \mathrm{C}$ for $2 \mathrm{~min}$. One millimolar EGTA was added, and the lysate was centrifuged at $1300 \mathrm{~g}$ for $7 \mathrm{~min}$. The supernatant was collected and removed. The nuclear pellet was washed in Solution A, resuspended in $100 \mu \mathrm{L}$ Solution B ( $3 \mathrm{mM}$ EDTA, $0.2 \mathrm{mM}$ EGTA), and allowed to incubate on ice for $10 \mathrm{~min}$. The tube was centrifuged at 10,000 $g$ for $7 \mathrm{~min}$, and the supernatant was collected as the soluble nuclear fraction. The pellet was resuspended in $100 \mu \mathrm{L}$ of modified RIPA lysis buffer (20 mM HEPES, $137 \mathrm{mM} \mathrm{NaCl}, 10 \%$ glycerol, $1 \%$ triton X-100, $0.5 \%$ sodium deoxycholate, $0.1 \%$ SDS, 2 mM EDTA) and incubated on ice for $10 \mathrm{~min}$. The tube was centrifuged at $1000 \mathrm{~g}$ for $10 \mathrm{~min}$, and the supernatant was collected as the chromatin fraction. $200 \mu \mathrm{L}$ of Laemmli buffer (Bio-Rad, Hercules, CA) was added directly to the remaining pellet for the insoluble fraction. Laemmli buffer was added to the cytoplasmic, soluble nuclear, and chromatin fractions at a 1:1 volume ratio. Phosphatase inhibitors (sodium orthovanadate, $\beta$-glycerophosphate, sodium pyrophosphate, sodium fluoride), SUMOylase inhibitor ( $N$-ethylmaleimide), $1 \mathrm{mM}$ DTT, and a complete mini EDTA-free protease inhibitor cocktail tablet (Roche Applied Science, Indianapolis, IN) were added to all fractionation buffers before use. The four fractions were resolved in NuPAGE Novex 4-12\% Bis-Tris precast gels (Invitrogen, Carlsbad, CA). Gels were stained with Coomassie blue to provide a general estimation of the protein lysate integrity. The gel lane from each fraction was sliced into ten equivalent sized bands (4 fractions, 10 slices $=40$ total reactions), and proteins were digested in-gel overnight with sequencing grade trypsin (Promega, Madison, WI).

\section{LC-MS/MS}

The extracted tryptic peptides from each of the 40 reactions were analyzed in duplicate by nanoflow reversed-phase liquid chromatography (LC) coupled online by electrospray ionization with a hybrid linear ion trap-Orbitrap MS (ThermoFisher Scientific, San Jose, CA). LC was performed using a Dionex Ultimate 3000 nanoflow LC system (Dionex Corporation, Sunnyvale, CA). Separation of the sample was performed using a $75 \mu \mathrm{m}$ inner diameter $\times 360$ outer diameter $\times 45 \mathrm{~cm}$-long fused silica capillary column (Polymicro Technologies, Phoenix, AZ) packed in house with a $5 \mu \mathrm{m}, 300$ Å pore size Jupiter C-18 stationary phase (Phenomenex, Torrance, CA). After injection, the column was washed with $98 \%$ mobile phase $\mathrm{A}(0.1 \%$ formic acid in water) for $30 \mathrm{~min}$ and peptides were eluted by development of a linear gradient of $2 \%$ mobile phase B $(0.1 \%$ formic acid in acetonitrile) to $42 \%$ mobile phase B in $140 \mathrm{~min}$, then to $98 \% \mathrm{~B}$ in an additional $20 \mathrm{~min}$, all at a constant flow rate of $250 \mathrm{~nL} / \mathrm{min}$. The MS was operated in a data-dependent MS/MS mode in which each full high resolution MS scan conducted in the Orbitrap was followed by seven MS/MS scans in the linear ion trap, where the seven most abundant peptide molecular ions dynamically determined from the MS scan were selected for tandem MS using a relative collision-induced dissociation (CID) energy of 30\%. Dynamic exclusion was utilized to minimize redundant selection of peptides for CID.

\section{Peptide Identification Using Inspect}

The spectra from each fraction (RIPA, S1, S3, pellet, and PreMN) were searched for peptide identifications by running Inspect against the human IPI protein database (v3.75) with parent mass tolerance $2 \mathrm{Da}$ and fragment mass tolerance $0: 5$. We allowed at most one modification $(\mathrm{K}+6, \mathrm{R}+10)$. To distinguish between the false and correct peptide identifications, the false discovery rate (FDR) approach using a standard target/decoy strategy was employed. Each Inspect search result was individually filtered at $1 \%$ FDR. 


\section{Estimation of Light to Heavy Ratio per Spectrum}

Quantification of protein abundance was achieved by measuring the relative intensities of light and heavy peptides in the MS spectra, which were spaced by $6 \mathrm{Da}$ for peptides with Lys $(\mathrm{K})$ or by $10 \mathrm{Da}$ for ones with Arg (R). Ratios were generated for each protein based on the corresponding peptide intensities (KU55933:vehicle) to determine the differential protein abundance in each fraction. Protein SILAC ratios were determined by averaging all peptide SILAC ratios from both unique and common peptides identified per given protein. Significance was assessed using a paired $t$ test which examines the shift in abundance level seen for all peptides annotated to a given protein before and after treatment. For the peptides that are identified by multiple spectra, we report a consensus ratio that is the ratio of the average light variant abundance to the average heavy variant abundance estimated across spectra. We also report consensus light to heavy ratio at protein level by averaging the estimated consensus log ratios of the peptides that uniquely match to the protein.

\section{Identification of Significantly Perturbed Subnetworks}

Protein interaction data was obtained from the Consensus-PathDB, which includes $\sim 100,000$ known protein-protein interactions compiled from a variety of interaction databases. Next, we applied the NeMo algorithm to this network to identify 2,507 densely connected subgraphs. Each subnetwork was then assessed for enrichment of proteins whose levels were significantly perturbed in the chromatin fraction using the hypergeometric cumulative distribution function:

$$
1-\sum_{i=0}^{k} \frac{\left(\frac{m}{i}\right)\left(\frac{N-m}{n-k}\right)}{\left(\frac{N}{n}\right)}
$$

where the four parameters $(k, m, n$, and $N)$ are defined as follows: $k$, the number of significantly perturbed proteins present in the subnetwork; $n$, the total number of proteins present in the subnetwork; $m$, the total number of significantly perturbed proteins; $N$, the total number of proteins identified in the chromatin fraction.

A significantly perturbed protein was defined as having either a $\log _{2}$ ratio $>0.58$ (a 1.5 -fold change in protein level) or a differential $p$-value $<0.05$.

\section{Network-Based Prioritization of Perturbed Proteins}

For each significantly perturbed protein in the chromatin fraction (defined as a $\log _{2}$ ratio $>0.58$ or a differential $p$-value $<0.05$ ), we examined its immediate neighbors in the protein interaction network for an enrichment of known DNA repair genes. The significance of enrichment was assessed using the hypergeometric CDF (formula given above) with the four parameters $(k, m, n$, and $N)$ defined as follows: $k$, number of neighbors in the protein interaction network annotated as DNA repair proteins; $n$, total number of neighbors in the protein interaction network; $m$, total number of proteins annotated as DNA repair proteins in the protein interaction network; $N$, total number of proteins present in the protein interaction network.

\section{Antibodies and Plasmids}

Antibodies used: mouse anti-ANXA1 and rabbit anti-p53 (Santa Cruz Biotech); mouse antiATM (Sigma); rabbit anti-phospho S1981 ATM (Epitomics); mouse anti-GAPDH, rabbit anti-histone $\mathrm{H} 3$, and mouse anti-lamin $\mathrm{A}+\mathrm{C}(\mathrm{Abcam})$; mouse anti-retinoblastoma (BD Biosciences); and mouse anti-53BP1 (Millipore). Lipofectamine 2000 (Invitrogen, Carlsbad, CA) was used for the transfection of plasmids into cells. ANXA-1 knockdown clones were 
established by lentivirus-mediated expression of an shRNA targeting

GCATTCTATCAGAA-GATGTAT.

\section{Live Cell Microscopy}

GFP-53BP1 expressing U2OS were selected using G418 $(50 \mu \mathrm{g} / \mathrm{mL})$ and plated on $35 \mathrm{~mm}$ round glass bottom dishes (MatTek Corp) $24 \mathrm{~h}$ prior to FRAP. U2OS were selected because they can be readily transfected. Cells were treated with $10 \mu \mathrm{M}$ KU55933 or DMSO at 15 min following 2 Gy IR. At +60 min post-IR, dishes were placed in an environmentally controlled closed chamber system (Tokai Hit) which was maintained at $37{ }^{\circ} \mathrm{C}$ and $5 \% \mathrm{CO}_{2}$ during imaging. The chamber system was mounted on an inverted confocal microscope (Nikon A1, Nikon) controlled by NIS-Elements software. At 75 min post-IR, time-lapse image acquisition was initiated on defined IR-induced 53BP1 foci to obtain baseline intensities. 53BP1 foci were photobleached using a 488 laser set at 100\% power, a scan speed of $1 / 8$ frames per second, and an exposure time of $53.57 \mathrm{~s}$, allowing seven laser pulses to hit a defined region in a single nucleus. Time-lapse images were captured for $30 \mathrm{~min}$ following photobleaching (from $+75 \mathrm{~min}$ to $+105 \mathrm{~min}$ following IR), in $30 \mathrm{~s}$ intervals with the 488 laser set at $5 \%$ power, a scan size of 1024 , a scan speed of $1 / 4$ frames per second, and a zoom of 1.499x. The excitation wavelength for GFP was a 488 laser, and the signal was collected through a CFI Plan Apo VC 60 Oil objective, NA = 1.40. A perfect focus system (PFS) was applied to automatically correct possible focus drift during the period of time-lapse imaging. Post-acquisition processing of image files was performed with NISElements software. We performed a double normalization to correct for acquisition bleaching for each ROI. The mean pre-bleach whole cell intensity of a neighboring cell expressing GFP-53BP1 was divided by the whole cell intensity of the neighboring cell at each time point in the post-bleach period, and was multiplied to the FRAP curve at that time point. To correct for background intensity, the mean intensity of a background ROI was subtracted from the neighboring cell ROIs and FRAP ROIs for each time point. Relative intensity (RI) was calculated by: $\mathrm{RI}(t)=I(t) / I_{\mathrm{pb}}$, where $I_{\mathrm{pb}}$ is the mean intensity of the ROI before photobleaching. Each data point was derived from the mean intensities of five different foci measurements from five different cells collected from three different experiments per condition.

\section{Clonogenic Survival Assays}

1,000 cells were seeded in $60 \mathrm{~mm}$ Petri dishes and treated with increasing doses of irradiation $4 \mathrm{~h}$ post-plating. $\mathrm{H} 460$ cells were selected because they are well-suited to clonogenic survival assays. $10 \mu \mathrm{M}$ KU55933 was added from $+15 \mathrm{~min}$ to $+75 \mathrm{~min}$ following IR. After 8-10 days, colonies were stained with crystal violet stain. A colony was defined as a cluster of $>50$ cells, presumably formed from a single cell, and all experiments were completed in triplicate. Error is reported as the standard error of the mean.

\section{RESULTS}

\section{Identification of Spatial Protein Dynamics Perturbed by KU55933 Using SILAC MS}

Two populations of primary human lung fibroblasts (IMR90) were grown in either "light" media ( ${ }^{12} \mathrm{C}_{6}$-lysine $/{ }^{12} \mathrm{C}_{6}{ }^{14} \mathrm{~N}_{4}$-arginine) or "heavy" media $\left({ }^{13} \mathrm{C}_{6}\right.$-lysine $/{ }^{13} \mathrm{C}_{6}{ }^{15} \mathrm{~N}_{4}$-arginine) to label proteins with isotopes that can be distinguished by MS (Figure 1A). ${ }^{14}$ Both populations of cells were exposed to 2 Gy IR, and cells grown in "heavy" media were treated with $10 \mu \mathrm{M}$ KU55933 from +15 to +75 min post-IR while cells grown in "light" media were treated with DMSO. Both populations were harvested and mixed at a 1:1 ratio, and four distinct subcellular fractions were generated, as evidenced by the immunoblot detection of the cytoplasmic protein GAPDH, the soluble nuclear protein retinoblastoma, the chromatin-bound protein histone $\mathrm{H} 3$, and the nuclear matrix/nuclear membrane-associated 
protein Lamin $\mathrm{A}+\mathrm{C}$ in fractionated lysates from IMR90 cells following IR (Supporting Information Figure 1B). The fractions were resolved, and each gel lane was cut into 10 pieces, digested with trypsin, and subjected to MS.

A total of 18,271 pairs of distinct light and heavy peptides were quantified (Supporting Information Table 1): 11,069 in the cytoplasmic, 8,591 in the chromatin, 4,607 in the soluble nuclear, and 6,796 in the insoluble pellet. Of these, 16,360 were unique peptides and 1,911 were common peptides (shared by more than 1 protein). The total number of proteins identified was 4,196: 2,992 in the cytoplasmic, 1,470 in the soluble nuclear, 2,240 in the chromatin, and 1,924 in the insoluble nuclear. The overlap in the shared number of proteins across the four fractions is depicted in Figure 1B. To identify proteins that significantly changed with KU55933, an MA plot was constructed for each fraction (Figure 1C; Supporting Information Figure 2A-C).

\section{Identification of DDR Proteins Perturbed by KU55933 in the Chromatin Fraction Using a DNA Repair Protein Network Analysis}

Challenged by such a large proteomic data set, we sought to identify whole pathways that are reconfigured in cells treated with KU55933 following IR. Analysis of gene expression data using Gene Set Analysis enrichment has facilitated the discovery of new groups of genes that are involved in specific biological functions. ${ }^{20}$ To this end we undertook an analogous approach and generated a protein interaction network from the ConsensusPathDB and applied the NeMo identification algorithm to isolate densely connected subnetworks that are often indicative of functional protein complexes. ${ }^{21}$ Each subnetwork was searched for an enrichment of proteins whose levels were significantly altered in the chromatin fraction. This analysis revealed 29 significantly enriched $(P<0.05)$ subnetworks (Figure 2; Supporting Information Table 2).

To refine our search, we decided to prioritize candidates based on associations with a biologically relevant network. We mined the chromatin fraction for proteins that changed with KU55933 and that were also enriched for direct interactions with a network of DNA repair proteins generated for this purpose $(n=436)$ (Supporting Information Table 3). These analyses identified 53BP1 as both differentially present in the chromatin and significantly enriched for interactions with DNA repair protein neighbors with a $p$-value of enrichment of $1.76 \times 10^{-27}$ (Supporting Information Table 4). The enrichment ratio for 53BP1 was 19.6 and $55.55 \%$ of its direct neighbors were DNA repair proteins. The association of 53BP1 with the chromatin fraction decreased by $19 \%$ with KU55933 $\left(\log _{2}\right.$ ratio $\left.=-0.3\right)$, and the $p$ value of this differential expression was 0.0007 . The differential abundance of 53BP1 in the chromatin fraction was the seventh most significant of 1,420 proteins analyzed, and the enrichment for DNA repair protein neighbors was the sixth most significant of 1,158 proteins analyzed (Figure 3A; Supporting Information Tables 1 and 4).

ANXA1 was also differentially present in the chromatin and significantly enriched for protein-protein interactions with DNA repair protein neighbors with a $p$-value of enrichment of $8.65 \times 10^{-4}$ (Figure 3A). The association of ANXA1 with the chromatin fraction decreased by $97 \%$ with KU55933 $\left(\log _{2}\right.$ ratio $\left.=-4.9\right)$, and the $p$-value of this differential expression was 0.002. The enrichment ratio for ANXA1 was 2.92, and $8.27 \%$ of its direct neighbors were annotated as DNA repair proteins. In a plot of all the proteins identified in both the cytoplasmic and chromatin fractions, ANXA1 protein levels in cells following IR do not change with KU55933 but are selectively decreased in the chromatin (Figure 3B). Furthermore, 53BP1 and ANXA1 are linked by USP28 in our DNA repair protein network (Figure 4). The differential abundance of ANXA1 in the chromatin fraction was the 13th most significant of 1,420 proteins analyzed, and the enrichment for DNA repair protein 
neighbors was the 102nd most significant of 1,158 proteins analyzed (Supporting Information Tables 1 and 4).

\section{Dynamic Exchange of GFP-53BP1 in DDR Complexes Is Decreased by KU55933}

Guided by the integrative network analysis, we pursued our observation that the retention of 53BP1 in the chromatin fraction decreased by $19 \%$ with KU55933. ATM kinase-dependent 53BP1 dynamics have not been detected previously. The accumulation of 53BP1 in DDR complexes is not ATM-dependent. ${ }^{22-24}$ Further, we have shown that ATM kinase inhibition for $1 \mathrm{~h}$ following IR affected neither the accumulation nor the resolution of 53BP1 in DDR complexes visualized in fixed cells. ${ }^{8}$

To validate our MS, we fractionated IMR90 cells treated with or without KU55933 following IR and immunoblotted 53BP1, the chromatin fraction. ImageJ quantification of three independent experiments showed a $28 \%$ reduction in 53BP1 in the chromatin fraction in cells treated with KU55933 (Figure 5A-C). To explain these observations, we hypothesized that KU55933 decreases the dynamic exchange of 53BP1 in DDR complexes, such that while the total number of 53BP1 protein complexes are the same, the amount of 53BP1 associated in each complex is decreased. To analyze the dynamics of GFP-53BP1 live cells following IR, FRAP was undertaken. U2OS cells transfected with GFP-53BP1 were treated with either DMSO or KU55933 from 15 min following IR. While $90 \%$ of GFP-53BP1 in foci were recovered in cells treated with DMSO, only 70\% of GFP-53BP1 in foci were recovered in cells treated with KU5593 (Figure 5D and E). Each data point was derived from the mean intensities of five different foci measurements from five different cells collected from three different experiments per condition. Thus, KU55933 disrupts the association of 53BP1 and perhaps DDR complexes in chromatin.

\section{ANXA1 Knockdown Sensitizes Cells to IR via a Mechanism that is not Potentiated by KU55933}

ANXA1 was both differentially present in the chromatin and significantly enriched for protein-protein interactions with DNA repair protein neighbors. Many functions of the annexins are linked to their ability to interact with cellular membranes in a reversible and regulated manner. ${ }^{12}$ ANXA1 is induced by heat, chemical, and oxidative stress, and has been implicated in the regulation of proliferation, differentiation, and apoptosis. ANXA1 has been detected in the nucleus of MCF7 cells after heat and knockdown of ANXA1 has been found to increase heat-induced DSBs that have been attributed to protein denaturation. ${ }^{13}$ Our MS indicates that ANXA1 is not an abundant protein in the chromatin fraction, and we have been unable to detect ANXA1 in the chromatin of H460, IMR90, or U2OS cells by immunoblotting. Since our methodology was developed to identify spatial protein dynamics with a greater sensitivity than can be attained by immunoblotting, we proceeded to investigate a functional role for ANXA1 in the DDR.

We generated clonal populations of $\mathrm{H} 460$ cells in which ANXA1 is knocked down by an shRNA and clonal H460 control cells expressing a scrambled shRNA (Figure 6A). No differences between the rate of proliferation of ANXA1 knockdown and control cells was observed. ANXA1 knockdown cells were radiosensitive relative to scrambled control cells (Figure 6B). The shoulder of the survival curves is the region where cells conduct repair of damage and are capable of surviving. The quasi-threshold dose, Dq, represents the sublethal dose and is $2.7 \mathrm{~Gy}$ for scrambled, 2.1 Gy for scrambled + KU55933, 1.2 Gy for ANXA1 knockdown, and 1.4 Gy for ANXA1 knockdown + KU55933. The radiosensitization observed in ANXA1 knockdown cells was not potentiated by KU55933. This suggests that ATM kinase and ANXA1 promote cell survival through a single mechanism during the $1 \mathrm{~h}$ window following IR. 


\section{CONCLUSIONS}

We combined SILAC MS/MS, a subcellular fractionation protocol, and network analyses to interrogate the dynamics of the cellular proteome that are dependent upon ATM kinase activity following IR. Integrating protein abundances and interaction networks enabled us to efficiently identify a small group of candidates in a way that has not before been possible.

These analyses revealed that the association of 53BP1 in the chromatin decreased with KU55933. This observation appeared to conflict with our previous observation that neither the accumulation nor the resolution of 53BP1 in DDR complexes visualized in fixed cells were affected by ATM kinase inhibition for $1 \mathrm{~h}$ following IR. ${ }^{8}$ However, FRAP analyses of live cells revealed that ATM kinase activity is essential for the dynamic exchange of 53BP1 in IR-induced DDR complexes. Thus, our analyses reveal the limitations of analyses of formalin fixed cells that fail to incorporate either temporal or dynamic analyses of protein complexes.

We hypothesize that ATM kinase inhibition disrupts the coordination of critical protein interactions and subsequent exchanges within DDR complexes that are required for appropriate repair. ATM kinase inhibition may cause DDR complexes to collapse, resulting in lesions that are masked from subsequent repair processes by residual proteins that remain bound at sites of DNA damage. Thus, ATM kinase inhibition may poison a subset of assembled DSB repair complexes. This model is consistent with our observations that ATM kinase inhibition for $1 \mathrm{~h}$ following IR increased persistent chromatid aberrations and increased cell death. ${ }^{7,8}$

We identified a novel role for ANXA1 in cellular radio-protection. Most annexin functions are associated with their ability to interact with cell membranes in a regulated and reversible manner. ${ }^{12}$ ANXA1 may be regulated following irradiation to promote cell survival. One intriguing possibility is that ANXA1, which has bivalent annexin activity, may link two or more membranes following irradiation to protect membrane trafficking and cell signaling. ANXA1 is also implicated in nuclear factor- $\kappa \mathrm{B}$ (NF- $\kappa \mathrm{B})$ activation through a direct interaction with NF- $\kappa$ B essential modulator (NEMO), the regulatory subunit of I $x$ B kinase (IKK). ${ }^{25} \mathrm{NF}-\kappa \mathrm{B}$, a transcription factor, modulates apoptotic responses induced by genotoxic stress. ATM phosphorylates NEMO following the induction of DSBs,${ }^{26}$ and it is possible that ANXA1 functions in an ATM kinase-dependent, NF- $\kappa$ B signaling pathway that is implicated in resistance to genotoxic therapy. ${ }^{27-31}$

The DNA repair neighborhood network analyses undertaken here revealed that ANXA1 interacts with DNA repair proteins. The strongest interactions identified were between ANXA1 and SLX1. SLX1-SLX4 is a structure specific endonuclease that resolves Holliday junctions. ${ }^{32,33}$ Disruption of the ATM-ANXA1-SLX1 interactions discovered here may explain the abrogation of SCE by ATM kinase inhibitors. ${ }^{8}$ It is possible that ATM kinase inhibition disrupts a functional interaction between ANXA1 and SLX4-SLX1 in chromatin, thereby preventing the formation of crossover products during HRR. The biological data supporting the interaction between ANXA1 and SLX1 was found in a proteomic identification of proteins that are copurified with SLX1. ${ }^{33}$ Thus, the network analyses described here are an excellent means to mine such supplemental data sets for significant interactions, to identify novel protein functions, and to generate new hypotheses.

\section{Supplementary Material}

Refer to Web version on PubMed Central for supplementary material. 


\section{Acknowledgments}

We thank Mark O'Connor for the KU55933, Jiri Lukas for the msGFP-53BP1 construct, and Lucas dos Santos and Han-Yu Chuang for help with network analyses. This work was funded in part by a John Lazo Molecular Pharmacology Training Fellowship grant (S.C.), CA148644 from the National Cancer Institute and the Frieda G. and Saul F. Shapira BRCA Cancer Research Program (C.J.B.), and grants ES014811 (T.I. and R.S.), ES019566 (B.V.H.) from the National Institute for Environmental Health Sciences, and P30CA047904.

\section{References}

1. Ciccia A, Elledge SJ. The DNA damage response: making it safe to play with knives. Mol Cell. 2010; 40:179-204. [PubMed: 20965415]

2. Savitsky K, Bar-Shira A, Gilad S, Rotman G, Ziv Y, Vanagaite L, Tagle DA, Smith S, Uziel T, Sfez S, Ashkenazi M, Pecker I, Frydman M, Harnik R, Patanjali SR, Simmons A, Clines GA, Sartiel A, Gatti RA, Chessa L, Sanal O, Lavin MF, Jaspers NG, Taylor AM, Arlett CF, Miki T, Weissman SM, Lovett M, Collins FS, Shiloh Y. A single ataxia telangiectasia gene with a product similar to PI-3 kinase. Science. 1995; 268:1749-1753. [PubMed: 7792600]

3. Matsuoka S, Ballif BA, Smogorzewska A, McDonald ER 3rd, Hurov KE, Luo J, Bakalarski CE, Zhao Z, Solimini N, Lerenthal Y, Shiloh Y, Gygi SP, Elledge SJ. ATM and ATR substrate analysis reveals extensive protein networks responsive to DNA damage. Science. 2007; 316:1160-6. [PubMed: 17525332]

4. Olsen JV, Vermeulen M, Santamaria A, Kumar C, Miller ML, Jensen LJ, Gnad F, Cox J, Jensen TS, Nigg EA, Brunak S, Mann M. Quantitative phosphoproteomics reveals widespread full phosphorylation site occupancy during mitosis. Sci Signal. 2010; 3:ra3. [PubMed: 20068231]

5. Bensimon A, Schmidt A, Ziv Y, Elkon R, Wang SY, Chen DJ, Aebersold R, Shiloh Y. ATMdependent and -independent dynamics of the nuclear phosphoproteome after DNA damage. Sci Signal. 2010; 3:rs3. [PubMed: 21139141]

6. Arlett CF, Harcourt SA. Survey of radiosensitivity in a variety of human cell strains. Cancer Res. 1980; 40:926-32. [PubMed: 7471106]

7. White JS, Choi S, Bakkenist CJ. Irreversible chromosome damage accumulates rapidly in the absence of ATM kinase activity. Cell Cycle. 2008; 7:1277-84. [PubMed: 18418045]

8. White JS, Choi S, Bakkenist CJ. Transient ATM kinase inhibition disrupts DNA damage-induced sister chromatid exchange. Sci Signal. 2010; 3:ra44. [PubMed: 20516478]

9. Bouwman P, Aly A, Escandell JM, Pieterse M, Bartkova J, van der Gulden H, Hiddingh S, Thanasoula M, Kulkarni A, Yang Q, Haffty BG, Tommiska J, Blomqvist C, Drapkin R, Adams DJ, Nevanlinna H, Bartek J, Tarsounas M, Ganesan S, Jonkers J. 53BP1 loss rescues BRCA1 deficiency and is associated with triple-negative and BRCA-mutated breast cancers. Nat Struct Mol Biol. 2010; 17:688-95. [PubMed: 20453858]

10. Bunting SF, Callen E, Wong N, Chen HT, Polato F, Gunn A, Bothmer A, Feldhahn N, FernandezCapetillo O, Cao L, Xu X, Deng CX, Finkel T, Nussenzweig M, Stark JM, Nussenzweig A. 53BP1 inhibits homologous recombination in Brcal-deficient cells by blocking resection of DNA breaks. Cell. 2010; 141:243-54. [PubMed: 20362325]

11. Polo SE, Jackson SJ. Dynamics of DNA damage response proteisn at DNA breaks: a focus on protein modifications. Genes Dev. 2011; 25:409-433. [PubMed: 21363960]

12. Gerke V, Creutz CE, Moss SE. Annexins: linking Ca2+ signalling to membrane dynamics. Nat Rev Mol Cell Biol. 2005; 6:449-61. [PubMed: 15928709]

13. Nair S, Hande MP, Lim LH. Annexin-1 protects MCF7 breast cancer cells against heat-induced growth arrest and DNA damage. Cancer Lett. 2010; 294:111-7. [PubMed: 20163912]

14. Mann M. Functional and quantitative proteomics using SILAC. Nat Rev Mol Cell Biol. 2006; 7:952-58. [PubMed: 17139335]

15. Ong SE, Mann M. Stable isotope labeling by amino acids in cell culture for quantitative proteomics. Methods Mol Biol. 2007; 359:37-52. [PubMed: 17484109]

16. Hickson I, Zhao Y, Richardson CJ, Green SJ, Martin NM, Orr AI, Reaper PM, Jackson SP, Curtin NJ, Smith GC. Identification and characterization of a novel and specific inhibitor of the ataxiatelangiectasia mutated kinase ATM. Cancer Res. 2004; 64:9152-9159. [PubMed: 15604286] 
17. Mendez J, Stillman B. Chromatin association of human origin recognition complex, cdc6, and minichromosome maintenance proteins during the cell cycle: assembly of prereplication complexes in late mitosis. Mol Cell Biol. 2000; 20:8602-8612. [PubMed: 11046155]

18. Smits VA, Reaper PM, Jackson SP. Rapid PIKK-dependent release of Chk1 from chromatin promotes the DNA-damage checkpoint response. Curr Biol. 2006; 16:150-159. [PubMed: 16360315]

19. Zou L, Cortez D, Elledge SJ. Regulation of ATR substrate selection by Rad17-dependent loading of Rad9 complexes onto chromatin. Genes Dev. 2002; 16:198-208. [PubMed: 11799063]

20. Begley TJ, Rosenbach AS, Ideker T, Samson LD. Damage recovery pathways in Saccharomyces cerevisiae revealed by genomic phenotyping and interactome mapping. Mol Cancer Res. 2002; 1:103-12. [PubMed: 12496357]

21. Rivera CG, Vakil R, Bader JS. NeMo: Network Module identification in Cytoscape. BMC Bioinformatics. 2010; 11(Suppl 1):S61. [PubMed: 20122237]

22. Schultz LB, Chehab NH, Malikzay A, Halazonetis TD. p53 binding protein 1 (53BP1) is an early participant in the cellular response to DNA double-strand breaks. J Cell Biol. 2000; 151:1381-90. [PubMed: 11134068]

23. Anderson L, Henderson C, Adachi Y. Phosphorylation and rapid relocalization of 53BP1 to nuclear foci upon DNA damage. Mol Cell Biol. 2001; 21:1719-1729. [PubMed: 11238909]

24. DiTullio RA Jr, Mochan TA, Venere M, Bartkova J, Sehested M, Bartek J, Halazonetis TD. 53BP1 functions in an ATM-dependent checkpoint pathway that is constitutively activated in human cancer. Nat Cell Biol. 2002; 4:998-1002. [PubMed: 12447382]

25. Bist P, Leow SC, Phua QH, et al. Annexin-1 interacts with NEMO and RIP1 to constitutively activate IKK complex and NF-kappaB: implication in breast cancer metastasis. Oncogene. 2011; 30:3174-3185. [PubMed: 21383699]

26. Wu ZH, Shi Y, Tibbetts RS, Miyamoto S. Molecular linkage between the kinase ATM and NFkappaB signaling in response to genotoxic stimuli. Science. 2006; 311:1141-1146. [PubMed: 16497931]

27. Criswell T, Leskov K, Miyamoto S, Luo G, Boothman DA. Transcription factors activated in mammalian cells after clinically relevant doses of ionizing radiation. Oncogene. 2003; 22:58135827. [PubMed: 12947388]

28. Huang TT, Wuerzberger-Davis SM, Wu ZH, Miyamoto S. Sequential modification of NEMO/ IKKgamma by SUMO-1 and ubiquitin mediates NF-kappaB activation by genotoxic stress. Cell. 2003; 115:565-576. [PubMed: 14651848]

29. Li N, Banin S, Ouyang H, et al. ATM is required for IkappaB kinase (IKKk) activation in response to DNA double strand breaks. J Biol Chem. 2001; 276:8898-8903. [PubMed: 11114307]

30. Piret B, Schoonbroodt S, Piette J. The ATM protein is required for sustained activation of NFkappaB following DNA damage. Oncogene. 1999; 18:2261-2271. [PubMed: 10327072]

31. Janssens S, Tschopp J. Signals from within: the DNA-damage-induced NF-kappaB response. Cell Death Differ. 2006; 13:773-784. [PubMed: 16410802]

32. Fekairi S, Scaglione S, Chahwan C, Taylor ER, Tissier A, Coulon S, Dong MQ, Ruse C, Yates JR 3rd, Russell P, Fuchs RP, McGowan CH, Gaillard PH. Human SLX4 is a Holliday junction resolvase subunit that binds multiple DNA repair/recombination endonucleases. Cell. 2009; 138:78-89. [PubMed: 19596236]

33. Svendsen JM, Smogorzewska A, Sowa ME, O'Connell BC, Gygi SP, Elledge SJ, Harper JW. Mammalian BTBD12/SLX4 assembles a Holliday junction resolvase and is required for DNA repair. Cell. 2009; 138:63-77. [PubMed: 19596235] 

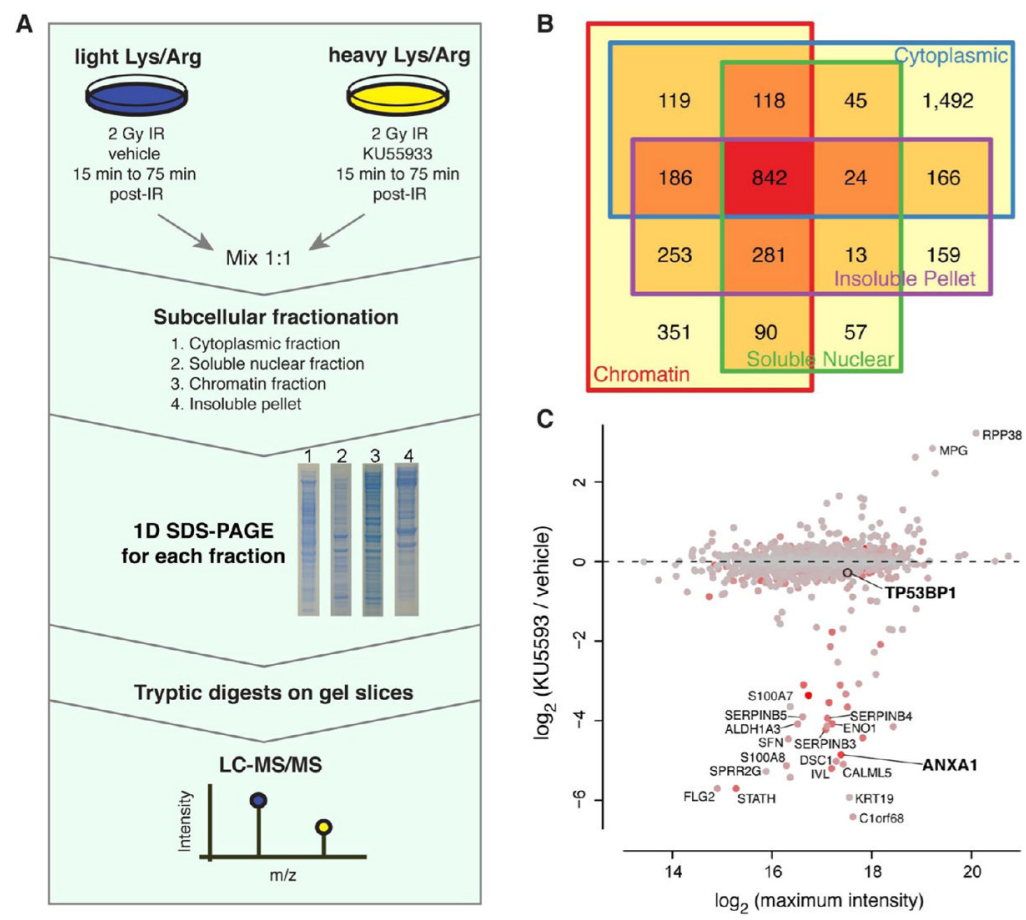

Figure 1.

(A) Cartoon depicting the SILAC workflow. (B) Overlapping proteins shared between subcellular fractions. (C) MA plots of proteins that changed with KU55933 in the chromatin fraction. The $y$-axis represents the differential expression of the protein with KU55933; the $x$-axis represents the relative abundance of the protein. A $t$ test was performed on the distribution of vehicle peptide intensities and KU55933 peptide intensities for each protein to generate a $p$-value for the differential expression of each protein. The pink-to-red color gradient used for the data points is a representation of the $p$-value: red indicates a low $p$ value. 


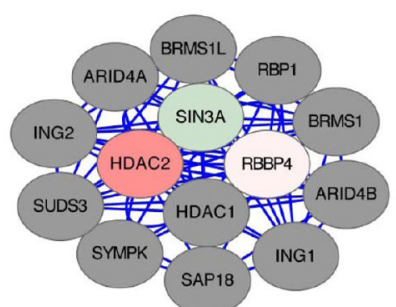

Chromatin remodeling

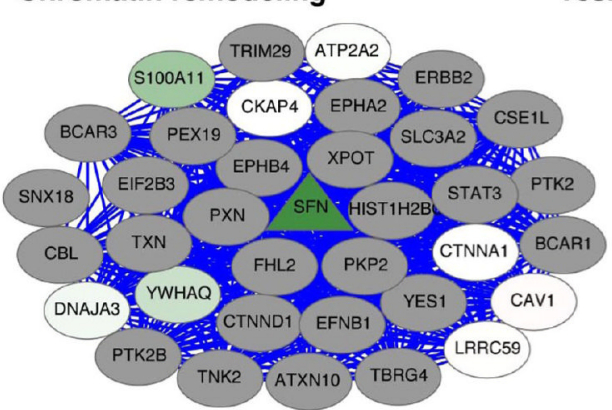

Protein tyrosine kinase signaling

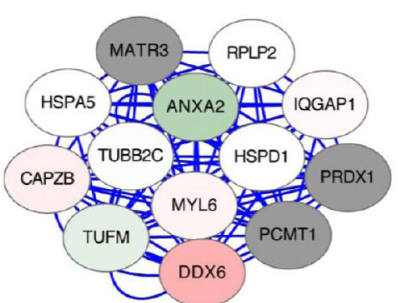

Vesicles

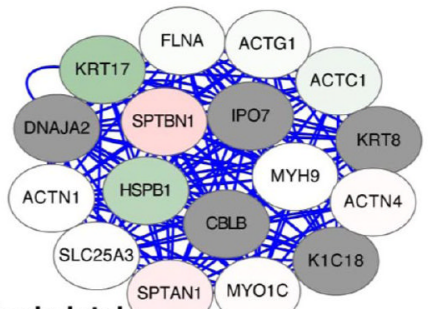

Cytoskeletal

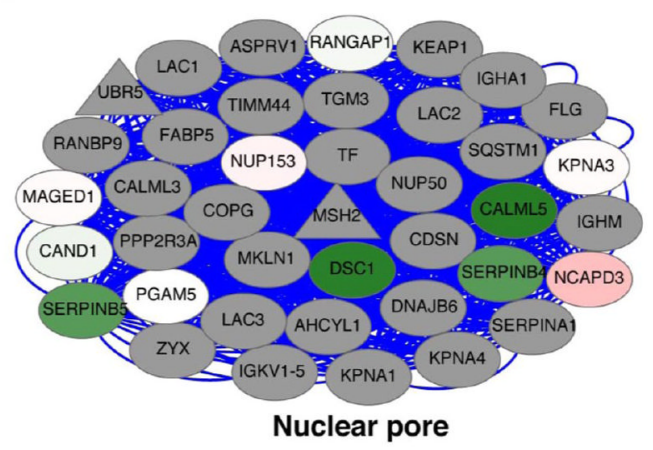

Figure 2.

Subnetworks significantly enriched with proteins that changed with KU55933. Triangles designate DDR proteins. Dark green indicates reduced protein abundance with KU55933; dark red indicates increased protein abundance with KU55933; gray indicates proteins not identified. 

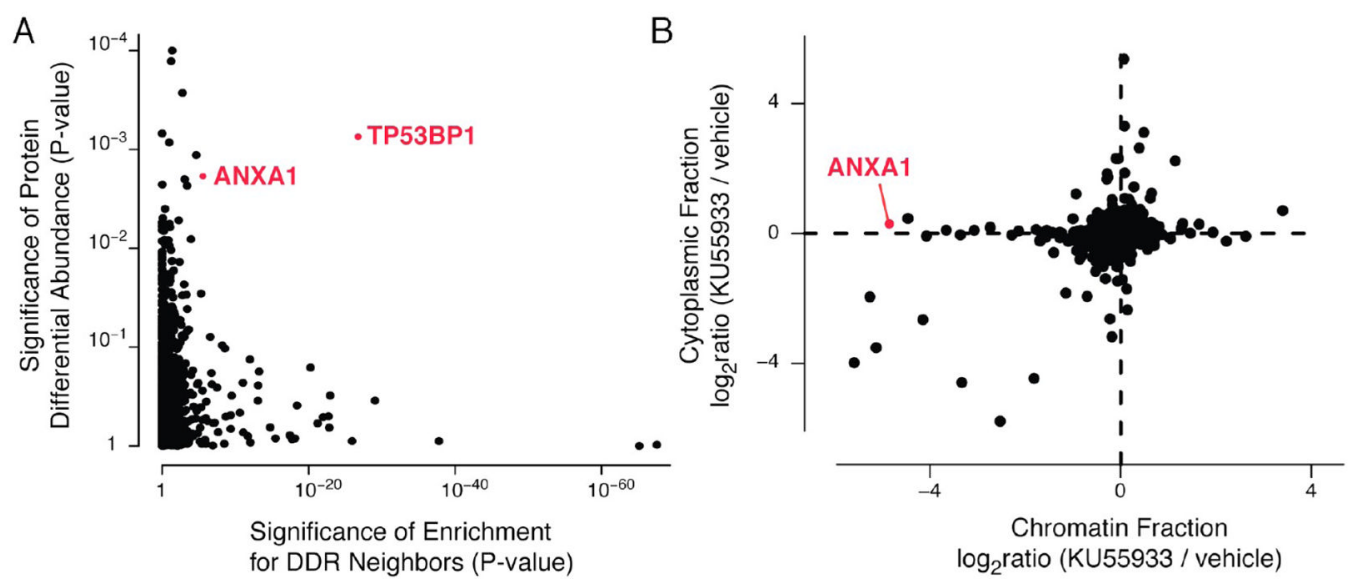

Figure 3.

(A) Comparison of the significance of protein abundance with the significance of protein enrichment for DDR neighbors. (B) Comparison of cytoplasmic with chromatin fractions for the differential expression of ANXA1 with KU55933 (53BP1 was not detected in the cytoplasm). 


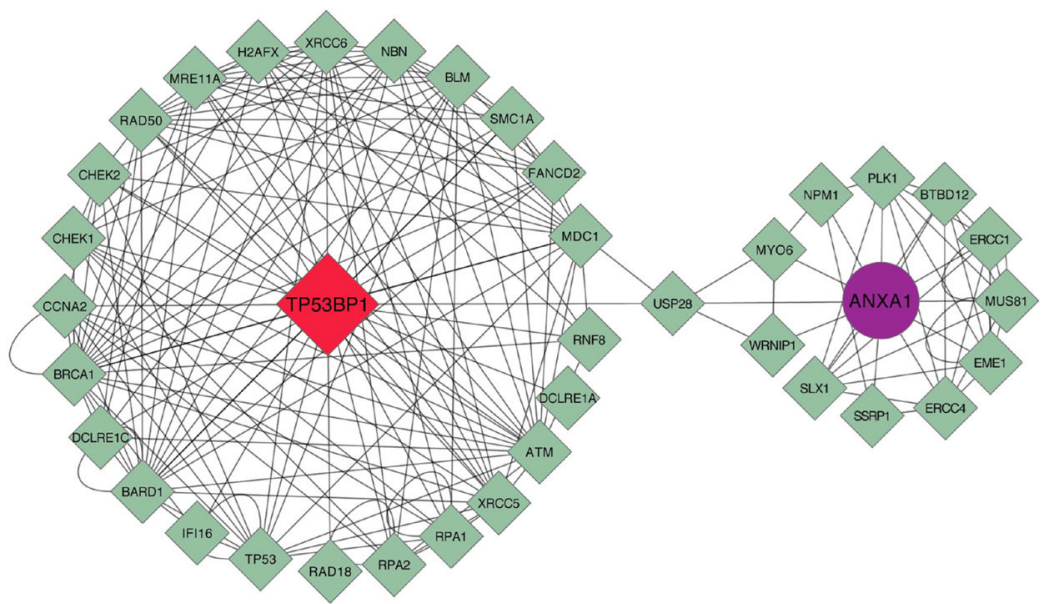

Figure 4.

53BP1/ANXA1 DNA repair protein subnetwork. The diamonds indicate known DDR proteins. 
A

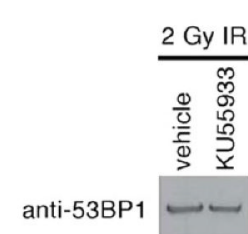

B

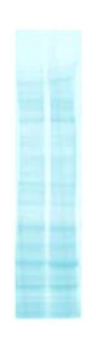

D

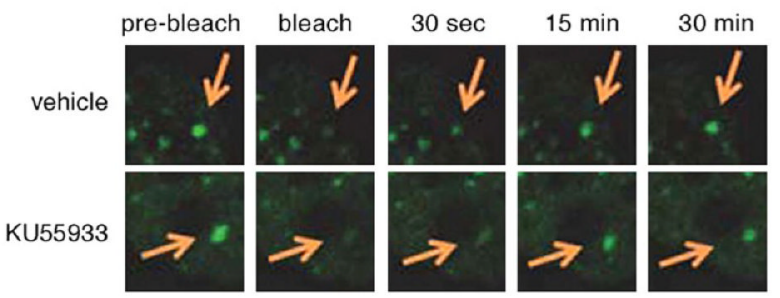

E

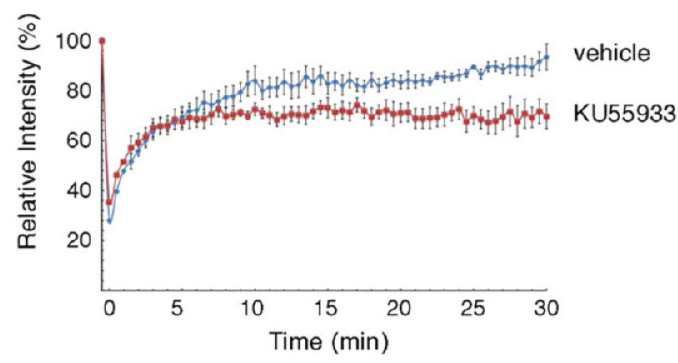

Figure 5.

(A) Immunoblot validation that 53BP1 is decreased in the chromatin fraction of IMR90 cells with KU55933. (B) Nitrocellulose filter stained with fast green. (C) 53BP1 is decreased by $28 \%$ in the chromatin fraction with KU55933. Error bars reflect the standard error of the mean from three separate experiments. (D) Representative time-courses of IR-induced GFP-53BP1 complex FRAP. (E) Relative fluorescence intensity curve plotted using the mean of five IR-induced GFP-53BP1 complexes, each in a different nucleus from five different cells derived from three different experiments. 
A

\begin{tabular}{|l|c|}
\hline Peptide & Ratio (H:L) \\
\hline K.GDRSEDFGVNEDLADSDAR.A & $1: 52$ \\
\hline K.GLGTDEDTLIEILASR.T & $1: 61$ \\
\hline R.SEDFGVNEDLADSDAR.A & $1: 47$ \\
\hline
\end{tabular}

B

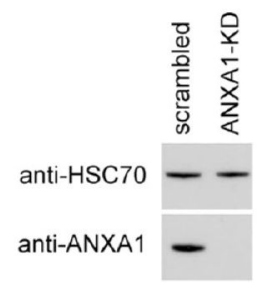

C

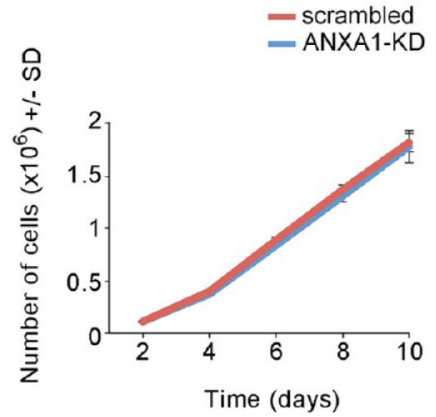

D

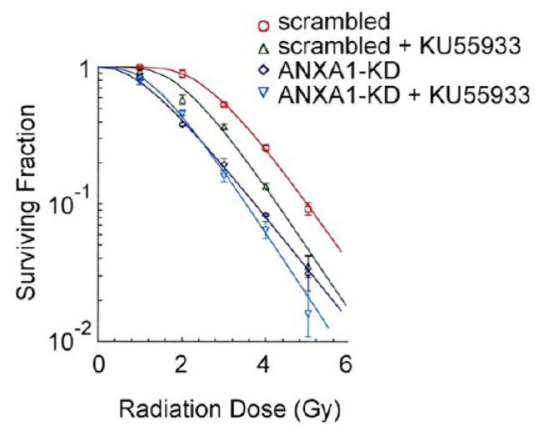

Figure 6.

(A) Unique ANXA1 peptides identified by SILAC. (B) ANXA1 knockdown (KD) in H460 cells. (C) Growth curves of ANXA1-KD and scrambled control H460 cells. (D) Clonogenic survival assays of ANXA1-KD and scrambled control H460 cells treated with and without KU55933 for $1 \mathrm{~h}$ following IR. 